VOL. 14 (1976), 267-278.

\title{
Representations of metabelian groups satisfying the minimal condition for normal subgroups
}

\section{Howard L. Silcock}

\begin{abstract}
A question of John $S$. Wilson concerning indecomposable representations of metabelian groups satisfying the minimal condition for normal subgroups is answered negatively, by means of an example. It is shown that such representations need not be irreducible, even when the group being represented is an extension of an elementary abelian p-group by a quasicyclic q-group of the type first described by V.S. Carin, and the characteristic of the field is a prime distinct from both $p$ and $q$. This implies that certain techniques used in the study of metabelian groups satisfying the minimal condition for normal subgroups are not available for the corresponding class of soluble groups of derived length 3 .
\end{abstract}

1.

In this paper we answer a question raised by Wilson in his talk [12] at the Mini-Conference on Group Theory at the Australian National University in November 1975.

Wilson's question arises in the context of a more general question, whether a soluble group that satisfies min-n (the minimal condition for normal subgroups) is necessarily countable. The latter question remains open [see note added in proof], though McDougall [6] has dealt with an important case by proving that all metabelian groups satisfying min-n are Received 16 December 1975. 
countable (see also [9]). By a result of Wilson [11], it is sufficient for the countability question to consider.soluble groups satisfying min-n that are also F-perfect: that is, have no proper subgroups of finite index. Further, it is easy to see that every group satisfying $\min -n$ is a subdirect product of a finite number of monolithic groups satisfying $\min -n$. (A group is monolithic if its non-trivial normal subgroups have non-trivial intersection.) Therefore, when it is convenient, we may restrict our attention further by considering only monolithic groups.

Detailed information about the structure of metabelian groups satisfying $\min -n$ has been obtained by Hartley and McDougall [2]. Indeed these authors have completely classified the $\underline{\underline{F}}$-perfect metabelian groups satisfying min-n. Their results imply, in particular, that a metabelian group which satisfies min- $n$ and is in addition both F-perfect and monolithic is very like one of the "standard" examples of metabelian groups satisfying min-n constructed by Carin [1]. For each pair of distinct primes $p$ and $q$, Carin's construction gives a group satisfying min-n which is an extension of an infinite elementary abelian $p$-group by an abelian group of type $q^{\infty}$. Following Wilson, we denote this group by $C\left(p, q^{\infty}\right)$.

Now suppose $G$ is a soluble group of derived length 3 which satisfies $\min -n$ and is both F-perfect and monolithic, and write

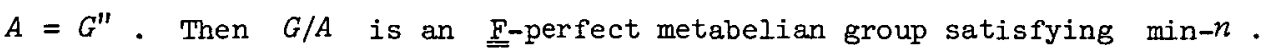
In attempting to prove that $G$ is countable it is natural, in view of the remarks made above, to consider first the special case where $G / A$ is actually isomorphic to $C\left(p, q^{\infty}\right)$, for some $p$ and $q$. Now $A$ is periodic (see Theorem 5.25 of [8]) and its non-trivial G-invariant subgroups intersect non-trivially because $G$ is monolithic. Therefore $A$ has only one primary component, and so is an $r$-group, for some prime $r$. Further, each of the subgroups

$$
A\left[r^{n}\right]=\left\{a \in A: a^{x^{n}}=1\right\},
$$

for $n=0,1,2, \ldots$, is $G$-invariant, and $G$ will be countable if and only if all the factors $A\left[r^{n}\right] / A\left[r^{n-1}\right]$ are countable. Thus we may further suppose that $A$ has exponent $r$.

After making the simplifications just indicated, we may view $A$ as a 
representation module for $C\left(p, q^{\infty}\right)$ over the field of $r$ elements $z_{p}$. The problem of describing $G$ thus leads naturally to the problem of describing representations of $c\left(p, q^{\infty}\right)$ over $Z_{p}$. This is the subject of Wilson's question, which is part of Problem 4 of his talk [see note added in proof]:

"Study irreducible and indecomposable modules over the fields of $r$ elements for Carin's group $C\left(p, q^{\infty}\right)$. If $r$ is distinct from $p$ and $q$, are monolithic modules necessarily irreducible?"

In the analogous situation for metabelian groups, all the monolithic modules which occur are irreducible, by a result of Kovács and Newman [4]. Nevertheless we shall show, by means of an example based on Neumann's example in [4], that Wilson's question has a negative answer. In fact, we shall prove:

THEOREM. Let $p$ and $q$ be distinct primes. If $r$ is a prime such that $r-1$ is divisible by $p$, then the Carin group $C\left(p, q^{\infty}\right)$ has a monolithic representation module over $z_{r}$ that is artinian but not irreducible.

\section{2.}

In our proof of the theorem we shall need to refer to a property of the Carin group $C\left(p, q^{\infty}\right)$ that does not seem to have been noted in the literature. To explain this property we first introduce some notation.

We assume that distinct primes $p$ and $q$ have been chosen once and for all, and we let $F$ be the field associated with $c\left(p, q^{\infty}\right)$. Thus $F$ is the field obtained from $Z_{p}$ (the field with $p$ elements) by adjoining a primitive $q^{n}$-th root of unity, $\omega_{n}$ say, for each positiv integer $n$. This field is the union of a tower of finite subfields

$$
F_{1} \subseteq F_{2} \subseteq F_{3} \subseteq \cdots
$$

where $F_{n}=Z_{p}\left(\omega_{n}\right)$, for each $n$. We write $\Omega$ for the subgroup of the multiplicative group of $F$ generated by $\omega_{1}, \omega_{2}, \ldots$; and we set $\Omega_{n}=\left(w_{n}\right)$, for $n=1,2,3, \ldots$.

LEMMA 1. There is a positive integer $d$ such that, for each integer 
$n \geq d$, the elements of any transversal to $\Omega_{n}$ in $\Omega$ form a basis for $F$ as a vector space over $F_{n}$.

Proof. Let $n$ be a positive integer and let $\Gamma$ be a transversal to $\Omega_{n}$ in $\Omega$. Since $F$ is generated as a field by $\Omega$, it is clear that the elements of $\Gamma \operatorname{span} F$ as a vector space over $F_{n}$. As a set is linearly independent if and only if every finite subset is linearly independent, it is sufficient to prove that, if $n$ is sufficiently large, the set $\Gamma \cap \Omega_{m}$ is linearly independent over $F_{n}$ for every $m>n$.

Now $\Gamma \cap \Omega_{m}$ is a transversal to $\Omega_{n}$ in $\Omega_{m}$, so it contains $\left|\Omega_{m} / \Omega_{n}\right|=q^{m-n}$ elements. These elements span $F_{m}$ as a vector space over $F_{n}$, so to show they are linearly independent it will be enough to prove that $\left|F_{m}: F_{n}\right|=q^{m-n}$.

By its definition, $F_{m}$ contains a primitive $q^{m}$-th root of unity and is the smallest field of characteristic $p$ with this property. So if $F_{m}=G F\left(p^{f}\right)$, then the order of the multiplicative group of $F_{m}$, namely $p^{f_{m}}-I$, must be divisible by $q^{m} ;$ and $f_{m}$ must be the least positive integer with this property. Thus $f_{m}$ is the order of $p$ modulo $q^{m}$, in the sense of number theory. Similarly $f_{n}$ is the order of $p$ modulo $q^{n}$. As $f_{m}=\left|F_{m}: Z_{p}\right|$ and $f_{n}=\left|F_{n}: z_{p}\right|$, we have

$$
\left|F_{m}: F_{n}\right|=f_{m} / f_{n}
$$

The result is therefore a consequence of the following simple numbertheoretic lemma.

LEMMA 2. Let $p$ and $q$ be distinct primes, and let $f_{n}$ be the order of $p$ modulo $q^{n}$, for $n=1,2,3, \ldots$. Let $d$ be the positive integer determined by the following conditions: 
(i) if $q$ is odd, or if $q=2$ and $p \equiv 1(\bmod 4)$, then $q^{d}$ is the largest power of $q$ dividing $p^{f_{1}}-1$;

(ii) if $q=2$ and $p \equiv 3(\bmod 4)$, then $2^{d}$ is the largest power of 2 dividing $p^{2}-1$.

Then, for all integers $m, n$ with $m>n \geq d$, we have

$$
f_{m} / f_{n}=q^{m-n}
$$

We omit the proof of this lemma, as the result may be deduced easily from Theorem 4-6 of [5] (p. 52).

We now consider the significance of Lemma 1 for the structure of $C\left(p, q^{\infty}\right)$. By definition, $C\left(p, q^{\infty}\right)$ is a split extension of the additive group of $F$ by the multiplicative group $\Omega$ defined above. To avoid the confusion caused by the simultaneous use of additive and multiplicative notations, we shall think of the elements of $C\left(p, q^{\infty}\right)$ as ordered pairs

$$
\left(\omega_{i}^{k}, \infty\right) \text {, }
$$

where $i, k$ are positive integers, and $\alpha \in F$. These pairs multiply according to the rule

$$
\left(\omega_{i}^{k}, \alpha\right)\left(\omega_{j}^{2}, \beta\right)=\left(\omega_{i}^{k} \omega_{j}^{2}, \alpha \omega_{j}^{2}+\beta\right) .
$$

Let us now write $b(\alpha)=(l, \alpha)$ and $c_{i}=\left(\omega_{i}, 0\right)$, for all $\alpha \in F$ and all $i>0$. Then, from (1), we have

$$
\begin{aligned}
& b(\alpha) b(\beta)=b(\alpha+\beta), \\
& c_{i}^{-1} b(\alpha) c_{i}=b\left(\omega_{i} \alpha\right),
\end{aligned}
$$

for all $\alpha, \beta \in F$ and all $i>0$. The elements $b(\alpha)$, for $\alpha \in F$, form a normal subgroup, $B$ say, of $C\left(p, q^{\infty}\right)$, and the mapping $b(\alpha) \mapsto \alpha$ is an isomorphism from $B$ onto the additive group of $F$. The subgroup $C$ generated by $c_{1}, c_{2}, \ldots$ is a complement to $B$, and the mapping $c_{i} \mapsto \omega_{i}$ evidently extends to an isomorphism between $C$ and $\Omega$. Further, $B$ is a minimal normal subgroup of $B C=C\left(p, q^{\infty}\right.$ ) (see, for example, $\$ 5.2$ of $[8]$ ). 
The following property of $B C$ turns out to be crucial for the construction to be described later.

LEMMA 3. There is a homomorphism $\pi$ from $B$ onto a finite direct factor $B_{0}$ of $B$ such that, for every $b \in B$, the set

$$
\left\{c \in C: b^{c} \pi \neq 1\right\}
$$

is finite.

Proof. Choose a positive integer $d$ satisfying the conditions of : Lemma 1 , and let $\Gamma$ be a transversal to $\Omega_{d}$ in $\Omega$ containing the identity element. Then Lemma 1 shows that $\Gamma$ is a basis for $F$ as a vector space over $F_{d}$, so $F$ is the direct sum of the subspaces $\gamma F_{d}$, as $\gamma$ ranges over $\Gamma$.

Now the mapping $\alpha \mapsto b(\alpha)$ is an isomorphism from the additive group of $F$ onto $B$, so this direct sum decomposition of $F$ determines a direct product decomposition of $B$, in which the summand $\gamma F_{d}$ of $F$ corresponds to the factor

$$
\left\{b(\gamma \alpha): \alpha \in F_{d}\right\}
$$

of $B$. Since $\Gamma$ contains the identity element, the subgroup

$$
B_{0}=\left\{b(\alpha): \alpha \in F_{d}\right\}
$$

occurs among these direct factors. Furthermore, if we set $t_{\gamma}=(\gamma, 0)$ for each $\gamma \in \Gamma$, then using equation (3) above we see that

$$
B_{0}^{t} \gamma=\left\{b(\gamma \alpha): \alpha \in F_{d}\right\}
$$

Consequently we can express $B$ as a direct product

$$
B=\operatorname{dr}_{\gamma \in \Gamma} B_{0}^{t} \gamma .
$$

Also, as the generators $c_{i}=\left(\omega_{i}, 0\right)$ of $C$ transform $B$ according to the mapping $b(\alpha) \mapsto b\left(\omega_{i} \alpha\right)$, the normalizer $N_{C}\left(B_{0}\right)$ is the finite subgroup $c_{0}=\left\langle c_{d}\right\rangle$ of $c$. But $C_{0}$ is the image of $\Omega_{d}$ under the 
isomorphism $\omega \mapsto(\omega, 0)$ between $C$ and $\Omega$, and this isomorphism maps $\Gamma$ onto the set

$$
T=\left\{t_{\gamma}: \gamma \in \Gamma\right\}
$$

Therefore $T$ is a transversal to $C_{0}$ in $C$.

Now define $\pi$ to be the canonical projection of $B$ onto $B_{0}$ associated with the direct decomposition (4). We claim that $\pi$ satisfies the conditions of the lemma. To see this, let $b \in B$. Then the projection of $b$ in the direct factor $B_{0}^{t}$ is trivial for all except a finite number of elements $t \in T$. Write $T_{0}$ for the finite subset of exceptional elements. Then, for any $c \in C$, the direct factors in which $b^{c}$ has non-trivial projection are precisely the factors $B_{0}^{t c}$, for $t$ ranging over $T_{0}$. In particular, the projection $b^{c} \pi$ of $b^{c}$ in $B_{0}$ is non-trivial if and only if $B_{0}^{t c}=B_{0}$, for some $t \in T_{0}$. This will be the case if and only if tc $\in N_{C}\left(B_{0}\right)=C_{0}$, for some $t \in T_{0}$. However, both $T_{0}$ and $C_{0}$ are finite, so there are only finitely many $c \in C$ such that $c \in t^{-1} C_{0}$ for some $t \in T_{0}$. Therefore $b^{c} \pi=1$ for all but a finite number of $c \in C$, and the result follows.

3.

We now come to the construction required to prove the theorem. We start by defining the conrestricted treble product, a variant of the treble product defined in [3].

Let $A, B, C$ be three groups and let

$$
\begin{aligned}
& \sigma: B \rightarrow \text { aut } A, \\
& \tau: C \rightarrow \text { aut } B
\end{aligned}
$$

be homomorphisms (where we write aut $X$ for the automorphism group of a group $X$ ). Using the homomorphism $\tau$, we first form the semi-direct product $H=B C$. Now write $P$ for the set of all functions from $C$ into $A$. Under the usual pointwise operations, $P$ is a group. We make $B$ act 
as a group of operators on $P$ as follows. If $f \in P$ and $h=b c_{1} \in H$, where $b \in B$ and $c_{1} \in C$, then we define $f^{h}$ by

$$
f^{h}(c)=f\left(c c_{1}^{-1}\right)^{b^{c^{-1}} \sigma}
$$

for all $c \in C$. It is a routine matter to check that the mapping $f \mapsto f^{h}$ is an automorphism of $P$ for each $h \in H$, and that the mapping which takes $h$ to the automorphism $f \mapsto f^{h}$ is a homomorphism from $H$ into aut $P$. We define the unrestricted treble product of $A, B, C$ (with associated homomorphisms $\sigma, \tau$ ) to be the semi-direct product $K=P H$, formed according to the action just described.

If $Q$ denotes the subgroup of $P$ consisting of all functions from $C$ into $A$ with finite support, then $Q$ is easily seen to be invariant under the action of $H$. The subgroup $T=Q H$ of $K$ is just the treble product

$$
\operatorname{tr}(A, B, C ; \sigma, \tau)
$$

defined in [3]. As usual, we may regard $A$ as a subgroup of $K$ by identifying each element $a \in A$ with the function $\hat{a}: C \rightarrow A$ defined by

$$
\begin{aligned}
& \hat{a}(c)=1, \text { if } c \neq 1, \\
& \hat{a}(1)=a .
\end{aligned}
$$

When this identification is made, it follows from (5) that $A$ is invariant under the action of $B$, and that $A B$ is isomorphic to the semi-direct product of $A$ and $B$ formed using $\sigma$.

In the terminology of Neumann [7], $K$ is an unrestricted twisted wreath product of $A$ and $H$, while $T$ is the corresponding restricted twisted wreath product.

We now consider the subgroup $M=\langle Q, D\rangle$ of $P$ obtained by adjoining to $Q$ the subgroup $D$ of all constant functions from $C$ into $A$. If $C$ is infinite, the elements of $D$ have infinite support, so that $D \cap Q=1$ and hence $M=D \times Q$. In general, $M$ need not be invariant under the action of $H$. However, we now show that if the homomorphisms $\sigma$ and $\tau$ are subject to certain conditions $M$ will be invariant.

LEMMA. 4. If $\sigma$ and $\tau$ are chosen so that, for each $b \in B$, the 
set $\left\{c \in C: b^{c} \sigma \neq 1\right\}$ is finite, then $M$ is invariant under the action of $H$. Furthermore, we have

$$
[M, H] \leq Q
$$

in this case.

Proof. As we know that $Q$ is $H$-invariant, it will be sufficient to prove that the conditions on $\sigma$ and $\tau$ imply that $[D, H] \leq Q$.

Let $f \in D$; then $f$ is a constant function, say $f(c)=a$, for all $c \in C$. If $h \in H$, then ve can write $h=b c_{1}$ for sultable $b \in B$ and $c_{1} \in C$, and then

$$
f^{h}(c)=f\left(c c_{1}^{-1}\right)^{b^{c^{-1}} \sigma}=a^{b^{c^{-1}} \sigma} .
$$

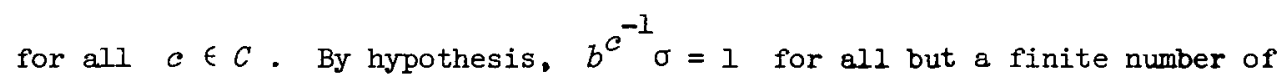
$c$, so $f$ and $f^{h}$ agree except on a finite subset of $C$. Therefore $[f, h]=f^{-I} f^{h}$ has finite support. Hence $[D, H] \leq Q$, and the result follows.

Now let $p, q, r$ be primes satisfying the conditions of the theorem. We specialize the construction just described, taking $B$ and $C$ to have the meanings ascribed to them in $\$ 2$, and choosing $\tau: C \rightarrow$ aut $B$ so that the associated semi-direct product $H=B C$ is the Carin group $C\left(p, q^{\infty}\right)$. For the group $A$ we take a cyclic group of order $r$. Then aut $A$ has order $r-I$, which by hypothesis is divisible by $p$; hence aut $A$ has a subgroup of order $p$.

Now let $\pi: B \rightarrow B_{0}$ be the homomorphism described in Lemma 3 . As $B_{0}$ is a non-trivial p-group, we can choose a non-trivial homomorphism $\sigma_{0}: B_{0}+$ aut $A$. We take $\sigma$ to be the homomorphism from $B$ into aut $A$ obtained by composing $\pi$ and $\sigma_{0}$.

With this choice of $\sigma$ and $\tau$, the treble product

$$
T=\operatorname{tr}(A, B, C ; \sigma, \tau)
$$

is of the type constructed in $\$ 4$ of [10]. Therefore, by Lemma 6 of [10], 
the normal subgroups of $T$ are well-ordered by inclusion; and, as $A$ has prime order, its normal closure $Q$ is contained in each non-trivial normal subgroup of $T$.

We now observe that the conditions of Lemma 4 are satisfied. For by our choice of $\sigma$ we have ker $\pi \leq \operatorname{ker} \sigma$; hence the set $\left\{c \in C: b^{c} \sigma \neq 1\right\}$ is contained in $\left\{c \in C: b^{c} \pi \neq 1\right\}$. But the latter is finite by Lemma 3. Hence Lemma 4 is applicable, and we conclude that $M=Q \times D$ is invariant under the action of $H$.

Now $M$ is isomorphic to a direct power of $A$, so it is an elementary abelian $r$-group. Hence $M$ may be viewed in a natural way as a representation module for $H$ over $Z_{r}$. From this point of view, the $H$-invariant subgroups of $M$ are just the submodules of $M$; and, as $Q$ is a minimal normal subgroup in $T=Q H$, it follows that $Q$ is an irreducible submodule of $M$. Further, $M / Q$ has order $r$ and is centralized by $H$, so it is a one-dimensional factor-module of $M$. Thus $M$ has composition length 2 , and so is certainly artinian. To complete the proof of the theorem, we need one more fact concerning $M$.

LEMMA 5. Q has no H-admissible complement in $M$.

Proof. Suppose on the contrary that $Q$ has an admissible complement $L$. Then $L$ is operator-isomorphic to $M / Q$, so it is a cyclic group of order $r$ and is centralized by $H$.

Suppose $f$ is a generator of $L$. Then for some $c \in C$ we have $f(c) \neq 1$. Write $f(c)=a$. Then, as $f$ commutes with $c$, we have

$$
f(c)=f^{c}(c)=f(1),
$$

so that $f(1)=a$. As $A$ has prime order, we have $A=\langle a\rangle$. Now choose $b \in B$ such that $b \notin$ ker $\sigma$. Then $a^{b \sigma} \neq a$. However, $f$ commutes with $b$, so

$$
a=f(1)=f^{b}(1)=f(1)^{b}=a^{b} .
$$

This contradiction completes the proof.

From this lemma it follows at once that $Q$ is contained in every nontrivial submodule of $M$. Consequently $M$ is a monolithic representation module for $H$; further $M$ is artinian and has a proper submodule $Q$. 
Hence the theorem is established.

Note added in proof, 5 March 1976. After this paper was accepted for publication the author learnt that $\mathrm{Dr} B$. Hartley has independently obtained much more general results on the problems discussed here, in a forthcoming paper. In particular he has shown that soluble groups satisfying min- $n$ can be uncountable.

The problem of Wilson's quoted in $\$ 1$ was taken from a draft version of [12]: however, the author understands that this version is now to be modified to take account of Hartley's results, and that the published version of [12] may no longer include this problem in the form stated here.

\section{References}

[1] В.С. Чарин [V.S. Carin], "Замечание об условин минимальности для подгрупп" [A remark on the minimal condition for subgroups], Dokl. Akad. Nauk SSSR (N.S.) 66 (1949), 575-576.

[2] B. Hartley and D. McDougalI, "Injective modules and soluble groups satisfying the minimal condition for normal subgroups", Bull. Austral. Math. Soc. 4 (1971), 113-135.

[3] H. Heineken and J.S. WiIson, "Locally soluble groups with min-n ", J. Austral. Math. Soc. 17 (1974), 305-318.

[4] L.G. Kovács and M.F. Newman, "Direct complementation in groups with operators", Arch. Math. (Basel) 13 (1962), 427-433.

[5] William Judson LeVeque, Topics in number theory, Vol. I (AddisonWesley, Reading, Massachusetts, 1956).

[6] David McDougall, "Soluble groups with the minimum condition for normal subgroups", Math. 2. 118 (1970), 157-167.

[7] B.H. Neumann, "Twisted wreath products of groups", Arch. Math. (Basel) 14 (1963), 1-6.

[8] Derek J.S. Robinson, Finiteness conditions and generalized soluble groups, Part 1 (Ergebnisse der Mathematik und ihrer Grenzgebiete, 62. Springer-Verlag, Berlin, Heidelberg, New York, 1972). 
[9] Howard L. Silcock, "Metanilpotent groups satisfying the minimal condition for normal subgroups", Math. 2. 135 (1974), 165-173.

[10] Howard L. Silcock, "On the construction of soluble groups satisfying the minimal condition for normal subgroups", Bull. Austral. Math. Soc. 12 (1975), 231-257.

[11] John S. Wilson, "Some properties of groups inherited by normal subgroups of finite index", Math. Z. 114 (1970), 19-21.

[12] John S. Wilson, "Locally soluble groups satisfying the minimal condition for normal subgroups", Proc. Mini-Conference Group Theory, Australian National University, Canberra, 1975 (Lecture Notes in Mathematics, to appear).

Department of Pure Mathematics, University of Adelaide, Adelaide, South Australia. 